\title{
Sind Antithrombotika bei Blutung im oberen Gastrointestinaltrakt unbedenklich?
}

\author{
Es ist naheliegend anzunehmen, dass \\ eine antithrombotische Therapie \\ den Verlauf einer oberen gastroin- \\ testinalen Blutung, die nicht durch \\ Varizen bedingt ist, ungünstig be- \\ einflusst. Doch eine Kohortenstudie \\ konnte jetzt keinen Zusammenhang \\ nachweisen.
}

— Aufgenommen in die Studie wurden 43 Patienten, die einen Vitamin-KAntagonisten erhielten, und 107 Patienten, die mit Thrombozytenaggregationshemmern behandelt wurden. Bei ihnen wurde der Verlauf einer oberen gastrointestinalen Blutung, die nicht durch Varizen bedingt war, dokumentiert und mit den Ergebnissen einer Kontrollgruppe von 242 Patienten ohne antithrombotische Medikation verglichen.

Insgesamt fand sich kein signifikanter Unterschied zwischen den verschiedenen Patientengruppen. Eine Re- zidivblutung trat in der Patientengruppe mit einem Vitamin-K-Antagonisten bei $11,6 \%$, unter Thrombozytenaggregationshemmern bei $7,5 \%$ und in der Kontrollgruppe bei 9,1\% auf. Auch bezüglich der Notwendigkeit für eine Notfalloperation ergab sich kein statistisch signifikanter Unterschied: 2,3\% bei Patienten mit Vitamin-K-Antagonisten, 1,9\% bei Patienten mit Thrombozytenaggregationshemmern und $1,2 \%$ in der Kontrollgruppe. Gleiches gilt für die Mortalitätsrate: $4,7 \%$ bei Patienten mit Vitamin-K-Antagonisten, 5,6\% bei Patienten mit Thrombozytenaggregationshemmern und 5,8\% in der Kontrollgruppe. Nur die Verweildauer im Krankenhaus war bei Patienten mit oraler Antikoagulation signifikant länger.

\section{Kommentar}

Diese Daten sind sicherlich überraschend. Allerdings muss man bedenken, dass im
Rahmen dieser Studie nicht das Risiko für eine obere gastrointestinale Blutung, sondern lediglich der Verlauf analysiert und verglichen wurde. Dass die komplexe moderne antithrombotische Therapie mit Thrombozytenaggregationshemmern und Vitamin-K-Antagonisten bei kardialen Patienten das Risiko für eine obere gastrointestinale Blutung im klinischen Alltag deutlich erhöht, wird jeder klinisch tätige Gastroenterologe bestätigen können. Dass allerdings der Verlauf der oberen gastrointestinalen Blutung nicht durch die antithrombotische Therapie beeinflusst werden soll, ist zumindest überraschend, um nicht zu sagen wenig glaubwürdig. Die Botschaft hör ich wohl, allein es fehlt der Glaube!

P. StiefelhageN =

- V. Oritz Bellver et al.

Outcome of non-varicel acute upper gastrointestinal bleeding in patients with antithrombotic therapy. Digestion 80 (2009) 89-94

\section{... und ein Satz dicker roter Ohren}

— Ein 63-jähriger Mann mit bekannter rezidivierender aseptischer Meningitis stellte sich erneut wegen Fieber und Kopfschmerzen vor. Bei leichter Nackensteifigkeit wurde der Liquor untersucht, in dem sich eine leichte monozytäre Pleozytose zeigte. Während der symptoma-

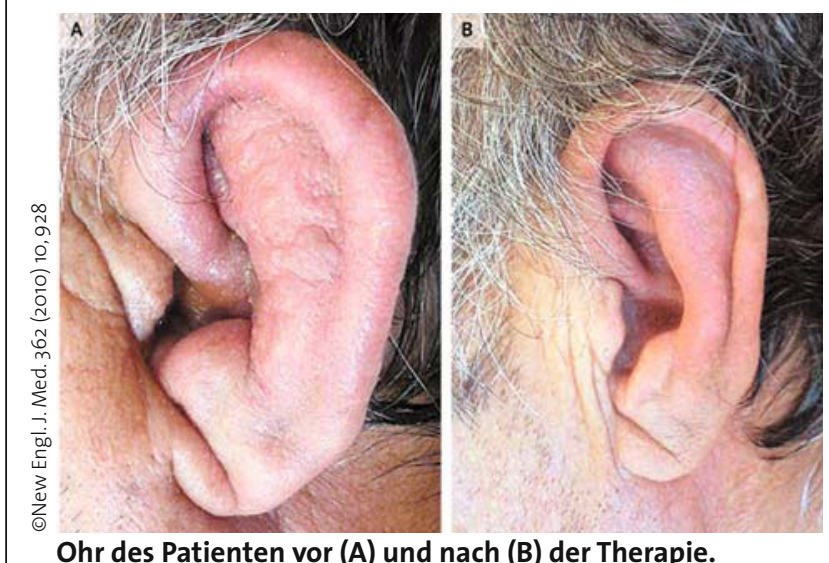

Ohr des Patienten vor (A) und nach (B) der Therapie. tischen Therapie entwickelte der Mann an beiden Ohren eine schmerzhafte Schwellung und Rötung (Abb. A). Eine Biopsie ergab entzündliche Veränderungen, die mit einer rezidivierenden Polychondritis vereinbar waren. Unter systemischer Steroidtherapie gingen die meningealen Symptome wie auch die Rötung und Schwellung der Ohren innerhalb weniger Tage zurück (Abb. B).

\section{Kommentar}

Bei der rezidivierenden Polychondritis ist in etwa $10 \%$ der Fälle mit ZNS-Symptomen zu rechnen. Möglicherweise lag bei dem Patienten bereits bei der ersten „Meningitis“ eine Polychondritis vor, es wurden aber die Rötungen im Bereich der Ohren nicht gesehen. Dass das nicht knorpelige Ohrläppchen von der Rötung ausgespart ist, zeigt deutlich, dass es sich hier um eine Knorpelentzündung handelt. H.-S. FüEßL =

- H. Kawai, A. Nakajima

(Shiga University of Medical Science Otsu, Japan, e-mail: hirok@ belle.shiga-med.ac.jp): Red puffy ears. New Engl. J. Med. 362 (2010) 10,928 